\title{
Le rôle clé de l'Assemblée citoyenne dans le débat sur l'avortement en Irlande
}

\section{Christophe Poiré}

\section{OpenEdition}

1 Journals

\section{Édition électronique}

URL : https://journals.openedition.org/etudesirlandaises/10292

DOI : 10.4000/etudesirlandaises. 10292

ISSN : 2259-8863

\section{Éditeur}

Presses universitaires de Caen

\section{Édition imprimée}

Date de publication : 31 décembre 2020

Pagination : 187-200

ISBN : 978-2-84133-996-9

ISSN : 0183-973X

\section{Référence électronique}

Christophe Poiré, «Le rôle clé de l'Assemblée citoyenne dans le débat sur l'avortement en Irlande », Études irlandaises [En ligne], 45-2 | 2020, mis en ligne le 31 décembre 2020, consulté le 14 novembre 2022. URL : http://journals.openedition.org/etudesirlandaises/10292 ; DOI : https://doi.org/10.4000/ etudesirlandaises. 10292

\section{(c) $)(7)(2)$}

Creative Commons - Attribution - Pas d'Utilisation Commerciale - Partage dans les Mêmes Conditions 4.0 International - CC BY-NC-SA 4.0

https://creativecommons.org/licenses/by-nc-sa/4.0/ 


\section{Le rôle clé de l'Assemblée citoyenne dans le débat sur l'avortement en Irlande}

Résumé: En 2016 fut instaurée en République d'Irlande une Assemblée citoyenne ayant pour objectif de réfléchir à divers enjeux sociétaux, tels que la légalisation de l'avortement, le réchauffement climatique, le vieillissement de la population et la réforme du Parlement... Le premier dossier sur lequel l'Assemblée dut se pencher fut celui de l'avenir du $8^{\mathrm{e}}$ amendement à la Constitution garantissant le droit à la vie de l'enfant à naître.

Le 25 mai 2018, le peuple irlandais se prononça en faveur de l'abrogation du $8^{\mathrm{e}}$ amendement qui avait été adopté trente-cinq ans plus tôt par 66,9\% de l'électorat. Le présent article a pour objectif, d'une part d'étudier le processus long et fastidieux qui déboucha sur la légalisation de l'avortement et, d'autre part d'analyser le rôle essentiel que joua l'Assemblée citoyenne dans ce résultat. Il semble fondamental de s'interroger sur l'autonomie politique de l'Assemblée. En quoi et comment parvient-elle à amoindrir la place de l'Église et de l'État dans le domaine de la morale sociale? Comment participe-t-elle à la mutation des mentalités et des comportements en République d'Irlande?

Mots clés: avortement, $8^{\mathrm{e}}$ amendement à la Constitution, Assemblée citoyenne, démocratie participative.

Abstract: In 2016, the Citizens' Assembly was established in the Republic of Ireland to consider some of the most important issues facing Ireland's future, such as abortion, climate change, ageing population and fixed term parliaments... The first question the Assembly had to discuss was the $8^{\text {th }}$ amendment to the Constitution which acknowledged the right to life of the unborn.

On 25 May 2018, 66.4\% of the electorate chose to delete the $8^{\text {th }}$ amendment which had been voted in by $66.9 \%$ of voters thirty-five years previously. The object of this paper is to study the long and tedious process of the legalisation of abortion in Ireland and to examine the decisive commitment of the Citizens' Assembly in the referendum result of May 2018. It is crucial to question whether or not the Assembly is an autonomous entity. Is it able to diminish the power of both the Church and state concerning moral issues, such as abortion? Is the Assembly a component of the change in the mentality of Irish people?

Keywords: abortion, $8^{\text {th }}$ amendment, Citizens' Assembly, deliberative democracy.

À l'automne 2016 s'est mise en place en République d'Irlande une Assemblée citoyenne consultative composée de cent membres, dont quatre-vingt-dix-neuf furent tirés au sort parmi la population irlandaise inscrite sur les listes électorales. Cette assemblée, toujours en place, a pour mission de réfléchir à l'évolution du pays dans des domaines variés, tels que la question de l'avortement, les effets sociaux du vieillissement de la population, l'instauration de mandats législatifs à durée fixe, la place du pays dans la lutte contre le changement climatique, les pratiques référendaires. 
Avec l'arrivée au pouvoir, en juin 2017, du nouveau Taoiseach, Leo Varadkar, qui considérait la loi en vigueur sur l'avortement comme trop restrictive, la tenue d'un référendum sur cette question fut envisagée pour 2018. Le premier chantier auquel l'Assemblée dut se consacrer fut donc celui de l'avenir du $8^{\mathrm{e}}$ amendement à la Constitution (de septembre 1983) qui reconnaissait alors le droit à la vie de «l'enfant à naître». Les conclusions et recommandations de l'Assemblée, dont le rôle ne fut cependant que consultatif, avaient pour objectif d'apporter des éclaircissements sur un sujet aussi complexe que clivant dans la société irlandaise. En effet, en 35 ans, de 1983 à 2018, non moins de six référendums furent organisés sur cette question dans un pays qui connaît, depuis l'adoption de la Constitution de 1937, une activité référendaire soutenue en raison de son caractère strictement obligatoire en matière de modification constitutionnelle. La multiplicité des consultations populaires sur l'interruption volontaire de grossesse (IVG) constitue donc une pratique courante, dans le même temps qu'elle traduit la difficulté à trouver une solution durable.

En remettant son premier rapport le 29 juin 2017 à une commission parlementaire, qui, elle-même, rendit ses conclusions au gouvernement en décembre 2017, l'Assemblée citoyenne occupa dès lors une place inédite dans la vie de la nation. Sa mise en place établit sans aucun doute un nouvel espace politique où la réflexion n'émana pas seulement du législateur mais provint de «tout un chacun». Après avoir contextualisé la situation, en analysant les éléments clés de l'histoire de l'avortement en Irlande, nous nous sommes interrogé sur cette forme actuelle de mobilisation des citoyens qui a permis une mise au jour des divers points de vue sur cette question controversée.

L'Assemblée citoyenne, dont le rôle fut décisif dans la nette victoire du "oui » en mai 2018, jouit-elle d'une autonomie politique? En quoi et comment parvientelle à amoindrir la place de l'Église et, dans une moindre mesure, celle de l'État, dans le domaine de la morale sociale ? Comment participe-t-elle à la mutation des mentalités et des comportements en République d'Irlande? En outre, l'Assemblée constitue-t-elle un élément de consolidation de la démocratie participative ou reste-t-elle malgré tout subordonnée au pouvoir politique en place?

Le vendredi 25 mai 2018, le peuple irlandais s'est prononcé à une écrasante majorité en faveur de l'abrogation de l'article 40.3.3 de la Constitution interdisant l'avortement. Avec un taux de participation de 64,13\%, le camp du «oui » a obtenu 1429981 suffrages $(66,4 \%)$ contre 723632 (33,6\%) aux partisans du statu quo.

Trente-cinq ans auparavant, lors du premier référendum de 1983 sur la question, la très catholique République d'Irlande était le seul pays de la Communauté européenne où l'IVG était totalement illégale. Il en était ainsi depuis une loi victorienne de 1861, l'Offences against the Person Act.

Lors de la consultation référendaire du 7 septembre 1983, les Irlandais approuvèrent largement l'amendement anti-avortement (le $8^{\mathrm{e}}$ amendement), puisque $66,9 \%$ des votants accordèrent leur suffrage au texte suivant: «L'État reconnaît le droit à la vie de l'enfant à naître. En tenant dûment compte du droit égal de la mère à la vie, il s'engage à respecter ce droit dans ses lois et, autant que possible, à défendre 
et favoriser ce droit par ses lois ${ }^{1}$. L'Église catholique et les partis conservateurs étaient donc parvenus à faire inclure, par référendum, cette interdiction dans la loi fondamentale.

Comme l'écrivait Chrystel Hug dans un article de 1994, «la victoire [de 1983] fut sans ambiguïté, même si la participation au vote ne fut que de 54,6\% $»^{2}$. En effet, une part importante de la population s'était abstenue de participer au scrutin. Cette faiblesse relative du taux de participation était-elle un premier signe avant-coureur du changement qui allait s'opérer plus de trois décennies plus tard? En tout cas, bon nombre de partisans de la libération des mœurs déduisirent des résultats du référendum de 1983 que certains catholiques se détachaient progressivement de la tutelle de l'Église. On peut voir dans cette forte abstention le début d'un processus, certes lent, correspondant à une nouvelle forme de mobilisation des militants antiamendement, réunis sous la bannière de l'Anti-Amendment Campaign (ACC).

Leur action présentait deux caractéristiques essentielles. D'une part, l'ACC constituait en elle-même un nouvel espace de réflexion plus ouvert, plus horizontal, où les militants n'hésitaient pas à remettre en cause les certitudes de l'Église et de l'État en matière de morale sociale. D'autre part, cette forme de rassemblement, dans un contexte où l'évocation même du mot "avortement» pouvait mettre mal à l'aise, y compris parfois chez les partisans du "non» au $8^{\mathrm{e}}$ amendement $^{3}$, allait se poursuivre de façon presque souterraine et ce, jusqu'au début des années 1990. En effet, bien que la diffusion d'informations sur l'avortement fût totalement illégale, le mouvement pro-choix, de manière souvent quasi clandestine, s'employait à assurer un rôle de conseil auprès des femmes qui souhaitaient en savoir davantage sur la maîtrise de leur grossesse. Pendant des années, chaque samedi, des militantes féministes du Women's Right to Choose Group ${ }^{4}$ n'hésitaient pas à distribuer, dans les centres commerciaux de Dublin, des tracts contenant un numéro de téléphone pour obtenir des conseils, voire un rendez-vous pour échanger sur les différentes options en cas de grossesse non désirée. Tout ceci se passait bien entendu en toute illégalité ${ }^{5}$. Ces femmes, militantes progressistes de gauche, montraient déjà des signes précurseurs de la manière de concevoir la politique différemment. En effet,

1. "The State acknowledges the right to life of the unborn and, with due regard to the equal right to life of the mother, guarantees in its laws to respect, and as far as practicable, by its laws to defend and vindicate that right" (les traductions sont les miennes).

2. Chrystel Hug, «L'avortement en Irlande», in Les Irlandaises: vers une reconnaissance, Paul Brennan, Richard Deutsch (dir.), Paris, Presses de la Sorbonne Nouvelle (L'Irlande politique et sociale; 5), 1994, p. 47.

3. Voir Mary Muldowney, "Pro-Choice Activism in Ireland since 1983 », Sexual Politics in Modern Ireland, Jennifer Redmond, Sonja Tiernan, Sandra McAvoy, Mary McAuliffe, Maria Luddy (dir.), Dublin, Irish Academic Press, 2015, p. 132. L'auteure de l'article insistait sur le fait que le Parti des travailleurs avait lancé en 1983 un tract appelant les électeurs à voter contre le $8^{\mathrm{e}}$ amendement à la Constitution, mais que le terme «avortement» n'y était pas employé et que, plus surprenant encore, le texte ne parlait pas des femmes.

4. Emily O’Reilly, «Referendum Gave Boost to Pro-Abortion Groups», Sunday Tribune, 2 octobre 1983, p. 1.

5. Mary Muldowney, «Pro-Choice Activism in Ireland since 1983 », p. 133. 
elles aspiraient à une société où les points de vue conventionnels des représentants de la hiérarchie catholique et des partis politiques seraient supplantés par d'autres, moins orthodoxes, émanant de simples députés ou sénateurs, d'élus de terrain ou de citoyens ordinaires engagés. On peut dire que les militants entraient en résistance contre les préceptes de l'Église et contre un État trop timide sur l'évolution des mœurs. Cette forme de rassemblement des citoyens ne préfigurait-elle pas d'autres formes actuelles de mobilisation?

Dans le camp des défenseurs de l'ordre moral, la campagne de 1983 avait été menée en mettant en avant des arguments faisant appel à l'émotion, aux inquiétudes, voire aux angoisses. Dans un cadre fortement marqué par les enseignements de l'Église et par son influence sur le législateur, les militants anti-avortement avaient pour mission de défendre avant toute chose l'enfant à naître. L'avortement étant gravement contraire à la loi morale catholique, voter «non » au référendum revenait à voter contre l'Église. Les militants anti-IVG, regroupés au sein de l'organisation Pro-Life Amendment Campaign (PLAC), avançaient même qu'un vote négatif pouvait être assimilé à un vote anti-irlandais ${ }^{6}$. L'Église s'était donc immiscée dans le débat politique par le biais de la PLAC mais aussi directement lorsque, par exemple, l'archevêque de Dublin avait fait lire aux fidèles de son diocèse une lettre circulaire appelant à voter «oui» lors de la messe précédant la consultation référendaire ${ }^{7}$. Pour mener une vie en accord avec la volonté de Dieu, l'électeur irlandais se devait de suivre les recommandations de l'Église. Comme le souligne Edwige Nault, «l'influence de la doctrine catholique sur la politique sociale est incontestable et marquée par les nombreuses références à la religion ${ }^{8}$. Edwige Nault consacre d'ailleurs, dans son ouvrage L'avortement en Irlande, tout un chapitre à "l'infiltration des valeurs catholiques dans les fonctions de l'État». En 1983, le mouvement pro-vie utilisa dans sa campagne anti-IVG cette proximité patente entre Église et État (que l'on retrouvait déjà lors de la rédaction de la Constitution de 1937) lorsqu'il propagea l'idée que la définition de l'identité irlandaise (Irishness) devait se confondre avec le concept théologique de droiture ${ }^{9}$. Trente-deux ans plus tard, en 2015, lorsque le gouvernement d'Enda Kenny entreprit de mettre en place une Assemblée citoyenne chargée d'étudier la question de l'avortement, le Taoiseach, aidé de son ministre de la Protection sociale, Leo Varadkar, n'usa-t-il pas de stratagèmes pour que le débat devienne un débat principalement politique, affirmant ainsi, de fait, une réelle séparation des sociétés civile et religieuse? L'Assemblée citoyenne permit de contourner l'Église et, par contrecoup, de donner du poids à de nouveaux référents, notamment l'État et le pouvoir politique en général et tout particulièrement le citoyen irlandais. En matière de droit familial, on tint de moins en moins compte du droit canon. Cette modification de l'attitude de l'État

6. Mary Muldowney, «Pro-Choice Activism in Ireland since 1983 », p. 130.

7. Edwige Nault, L'avortement en Irlande: 1983-2013. Dimensions religieuses, socioculturelles, politiques et européennes, Francfort, P. Lang, 2015, p. 87-88.

8. Ibid., p. 84.

9. Mary Muldowney, «Pro-Choice Activism in Ireland since 1983 », p. 130. 
irlandais trouve sans aucun doute ses racines dans l'histoire même des questions de société, telles que la contraception, l'avortement, le divorce ou l'homosexualité.

En 1992, le cas X bouleversa le pays entier et mobilisa le camp libéral qui, le 17 février, organisa à Londres et à Dublin deux grandes manifestations pour condamner un jugement de la Haute Cour interdisant à une jeune fille, dite X, de se rendre en Angleterre pour subir un avortement. Cette dernière, alors âgée de quatorze ans, était tombée enceinte à la suite de viols commis par un proche de son père ${ }^{10}$. Prévenue trop tard de la décision judiciaire, la famille catholique pratiquante, qui se trouvait déjà en Angleterre, respecta le jugement temporaire de la Haute Cour et retourna en Irlande sans que l'adolescente ait subi une IVG. On voit ici comment cette décision joua sur la double culpabilité de l'adolescente et de sa famille: celle de vouloir avorter et celle de commettre un acte hors la loi qui transgressait la norme morale ${ }^{11}$. Comme le souligne Chrystel Hug dans The Politics of Sexual Morality in Ireland, le verdict fut accueilli avec effroi par une majorité d'Irlandais ${ }^{12}$ et un sondage, effectué quelques jours après la décision du juge Costello, révéla que $64 \%$ des personnes interrogées (contre $25 \%$ ) désapprouvaient celle-ci. $66 \%$ des sondés ( $28 \%$ y étaient opposés) déclaraient même être favorables à une révision du $8^{\mathrm{e}}$ amendement à la Constitution qui autoriserait alors une IVG dans des circonstances clairement définies et limitées, comme à la suite d'un viol par exemple.

Les 24 et 25 février 1992, les parents, avec l'aide des services gouvernementaux, firent appel du jugement auprès de la Cour suprême qui, après trois jours de débat à huis clos, cassa le jugement de la Haute Cour et autorisa l'adolescente à se rendre en Angleterre. Quatre juges sur cinq conclurent que, en cas de danger «réel et substantiel » pour la vie de la mère, une IVG pouvait être autorisée ${ }^{13}$. L'avortement devint alors, de facto, partiellement légal pour les Irlandaises sous réserve qu'il fût pratiqué hors du pays ${ }^{14}$. Ce verdict historique fit jurisprudence les années suivantes.

Afin de régler la situation délicate des quelque cinq mille femmes qui, chaque année, sortaient clandestinement du pays pour obtenir une IVG, le Premier ministre Albert Reynolds prit l'initiative, en novembre 1992, d'une consultation référendaire. En proposant aux Irlandais de s'exprimer à nouveau sur l'avortement, l'État prit le pas sur la hiérarchie catholique et écouta les aspirations d'une partie grandissante

10. Le 5 février 1992, la Haute Cour rendit un jugement temporaire empêchant la jeune fille de gagner l'Angleterre. Le 10 février se tint, à huis clos, l'audience des parents auprès du tribunal et, sept jours plus tard, le président de la Haute Cour, le juge Costello, confirma le jugement temporaire. L'adolescente ne pouvait se rendre en Angleterre pour une durée de neuf mois et ne pouvait donc avoir recours à un avortement.

11. Chrystel Hug, «L'avortement en Irlande», p. 55.

12. Chrystel Hug, The Politics of Sexual Morality in Ireland, Londres - New York, Macmillan St. Martin's Press, 1999, p. 167.

13. Center for Family and Human Rights, «Irlande: un ministre de la Santé veut furtivement légaliser l'avortement par décret», 19 octobre 2012, en ligne: https://c-fam.org/friday_fax/irlande-unministre-de-la-sante-veut-furtivement-legaliser-1-avortement-par-decret-3782.

14. Geraldine Kennedy, "Abortion Is Legal in Limited Cases, Says Supreme Court», The Irish Times, 6 mars 1992, p. 1. 
de la population qui remettait en cause les enseignements de l'Église. Le peuple irlandais fut appelé à se prononcer non pas par un, mais par trois référendums visant à réviser l'article 40.3.3 de la Constitution en y ajoutant trois dispositions distinctes. La première concernait la liberté de déplacement entre l'Irlande et un autre État ${ }^{15}$, la deuxième portait sur le droit à l'information au sujet de services légalement disponibles dans un autre État ${ }^{16}$ et la troisième envisageait une légalisation partielle de l'avortement lorsqu'une IVG était nécessaire pour sauver la vie (et pas simplement la santé) de la mère, à l'exclusion du risque d'autodestruction (c'est-à-dire du suicide) ${ }^{17}$.

Le mercredi 25 novembre 1992, le peuple se rendit aux urnes afin de voter sur les trois questions. Les propositions portant sur la liberté de voyager et sur la diffusion d'informations furent adoptées avec respectivement $62,3 \%$ et 59,9\% des suffrages exprimés. Mais l'amendement (le $12^{\mathrm{e}}$ ) sur la légalisation partielle de l'avortement en cas de danger pour la vie de la mère n'obtint que 34,6 \% de «oui». Comment comprendre que les électeurs aient massivement rejeté cette proposition cruciale?

Il est vrai que le troisième alinéa était très ambigu. D’ailleurs, comme le soulignait Chrystel Hug, au moment de la campagne,

Les Irlandais commencèrent [...] à suivre avec effroi et confusion les innombrables débats hyper-médiatisés, au cours desquels ils se rendirent compte que le Premier ministre et le ministre de la Santé, entre autres, ne parvenaient pas à se mettre d'accord sur le sens des mots insérés dans le $12^{\mathrm{e}}$ amendement tel qu'il était proposé, et se contredisaient les uns les autres ${ }^{18} \ldots$

Ces référendums très alambiqués amenèrent, notamment en ce qui concerne le troisième libellé, les défenseurs du droit à la vie et des partisans de la légalisation, qui jugèrent la formulation trop restrictive, à faire le même choix politique, c'està-dire celui du «non ${ }^{19}$. Le résultat montra que cette consultation référendaire, organisée près de dix ans après le référendum de 1983 et seulement sept mois après l'affaire $\mathrm{X}$, avait pâti de deux défauts: le manque de rigueur du pouvoir politique dans la campagne, le manque de clarté du texte du $12^{\mathrm{e}}$ amendement.

Le gouvernement Fianna Fáil d'Albert Reynolds s'était donc lancé, à la suite de l'affaire $\mathrm{X}$, dans une campagne insuffisamment préparée alors que, comme le

15. Le texte de la première disposition était le suivant: "This subsection shall not limit freedom to travel between the State and another state».

16. Le libellé de la deuxième disposition était le suivant: "This subsection shall not limit freedom to obtain or make available, in the State, subject to such conditions as may be laid down by law, information relating to services lawfully available in another state».

17. Le texte de la troisième disposition était le suivant: «It shall be unlawful to terminate the life of an unborn unless such termination is necessary to save the life, as distinct from the health, of the mother where there is an illness or disorder of the mother giving rise to a real and substantial risk to her life, not being a risk of self-destruction".

18. Chrystel Hug, «L'avortement en Irlande», p. 55.

19. Christophe Poiré, La question du divorce en Irlande (1983-1995), thèse de doctorat en études irlandaises, université d'Artois, 2000, p. 282-284. 
souligne Edwige Nault, «l'imbroglio juridique créé par X aurait dû inciter l'exécutif à s'orienter vers un projet de loi permettant de clarifier la situation ${ }^{20}$. Comme on avait privilégié le référendum par rapport à la voie parlementaire, les acteurs de la campagne ne furent pas seulement les partis politiques, fort divisés d'ailleurs sur la question, y compris en interne. En effet, les groupes de pression pro-vie et pro-choix, l'Église catholique (qui d'ailleurs ne soutint pas le Fianna Fáil, son allié politique habituel), les églises protestantes, les syndicats, certaines organisations professionnelles et d'autres groupes d'intérêt participèrent à la campagne. La multiplicité des points de vue rendit la question posée encore plus complexe à appréhender. Il semble patent que le manque d'explications et de pédagogie dans la campagne fut un frein à la prise de conscience de l'enjeu sociétal du projet gouvernemental, d'autant plus que la consultation référendaire se tenait le jour même d'élections législatives anticipées à la suite de la chute du gouvernement de coalition $^{21}$. Pendant la campagne, le gouvernement Fianna Fáil de Reynolds, seul à soutenir le «oui» aux trois questions posées, n'avait guère fait preuve de sens pédagogique. Comme l'indique Francis Hamon, « une campagne référendaire doit être didactique $»^{22}$.

La question de l'avortement revint sur le devant de la scène en 2002, dix ans après l'affaire X. Le gouvernement Fianna Fáil, avec le soutien de l'Église, souhaita, au motif de «clarifier la jurisprudence résultant de l'affaire $\mathrm{X}{ }^{23}$, durcir la loi sur l'avortement. Un cinquième référendum fut alors organisé. Le 6 mars 2002, le résultat du scrutin, dont le taux de participation n'atteignit pas les $50 \%$, fut très serré: 50,42 \% se prononcèrent contre le durcissement de la législation. Les Irlandais n'avaient pas suivi les consignes de vote de l'Église et du gouvernement Fianna Fáil.

Les promoteurs de la campagne anti-avortement eurent recours à des moyens de communication on ne peut plus conventionnels (conférences de presse, meetings, débats, affiches publicitaires...) qui mettaient davantage en avant le positionnement de l'autorité de l'État et de l'Église (et donc essentiellement des hommes) que la position de la femme dans la société et son droit à disposer de son propre corps. La campagne référendaire de 2002 légitimait le rapport de domination d'une minorité (le personnel politique et les membres influents de l'Église) sur une majorité (les citoyens et notamment les citoyennes). Ce référendum allait finalement davantage sanctionner les pouvoirs en place que fournir une réponse claire à la question posée. Fintan O’Toole, l'éditorialiste de The Irish Times, vit dans ce vote négatif un signe de rupture, de mutation des comportements entre 1983 et 2002 car «jamais auparavant, l'électorat n'avait osé se rebiffer contre l'union sacrée de Rome et du nationalisme irlandais ${ }^{24}$. Les tergiversations de l'État à prendre en main la

20. Edwige Nault, L'avortement en Irlande: 1983-2013..., p. 90.

21. Robert Henry, "The 25 November 1992 Referendum and General Election in the Republic of Ireland ", Living Archives, no 4, 1992, p. 78.

22. Francis Hamon, Le référendum: étude comparative, Paris, LGDJ-Lextenso, 2012, p. 40.

23. Edwige Nault, L'avortement en Irlande: 1983-2013..., p. 91.

24. Fintan O’Toole, "No Longer Yielding to Party or Pulpit, Electorate not Bashful of Uncertainty", The Irish Times, 8 mars 2002, p. 14. 
question, alors que deux rapports (ceux du Constitution Review Group de 1996 et du All-Party Oireachtas Committee on the Constitution de 2000) et un livre vert (Green Paper on Abortion de 1999) furent publiés, montrèrent, selon Edwige Nault, que «seules les affaires ayant un caractère médiatique [pouvaient] motiver un gouvernement à examiner la question $»^{25}$.

Un événement malheureux, le décès de Savita Halappanavar, jeune femme d'origine indienne de 31 ans morte à l'hôpital universitaire de Galway, après s'être vu refuser un avortement alors qu'elle faisait une fausse couche, raviva le débat sur l'IVG en octobre 2012. Rappelons que, malgré le jugement de la Cour suprême dans l'affaire X qui autorisait l'avortement en cas de danger pour la vie de la mère, aucune loi, qui aurait permis de faire appliquer la décision judiciaire, n'avait été adoptée par le Parlement irlandais. Le médecin de Savita Halappanavar avait refusé de pratiquer une IVG car, d'après lui, «tant qu'il y [avait] un rythme cardiaque fotal, [il] ne [pouvait] rien faire». Une sage-femme rappela d'ailleurs à la jeune Indienne qu'elle se trouvait dans un pays catholique. Quelques jours plus tard, le 28 octobre 2012, une semaine après son admission à l'hôpital, la patiente mourait de septicé$\mathrm{mie}^{26}$. Plusieurs manifestations eurent lieu dans le pays pour rendre hommage à la jeune femme et l'État fut accusé de ne pas prendre ses responsabilités politiques. Le Taoiseach Enda Kenny, bien que catholique pratiquant et opposé à l'avortement sur demande, se vit dans l'obligation d'appréhender la situation délicate dans laquelle le pays se trouvait, d'autant plus que, depuis novembre 2007, le Commissaire aux droits de l'homme du Conseil de l'Europe avait fortement préconisé de mettre la législation en conformité avec le jugement de la Cour suprême.

En quelques années, par les voies parlementaire et référendaire, la question de l'avortement connut des avancées significatives. Si, en 1992 comme en 2002, le manque de temps et de maturité avait prévalu, le processus lent mis en œuvre de 2015 à 2018 permit en revanche de faire face au malaise qui rongeait la société irlandaise depuis des décennies.

Le 12 juillet 2013, après que plusieurs commissions de groupes d'experts eurent donné leur avis sur l'opportunité de privilégier la voie parlementaire sur la voie référendaire, le Dáil adopta avec 127 voix pour, 31 contre et 3 abstentions le projet de loi intitulé Protection of Life during Pregnancy Bill 2013 ${ }^{27}$. Promulguée par le président de la République le 30 juillet, après son adoption à une majorité claire par le Sénat (39 voix pour, 14 voix contre et 6 abstentions), la nouvelle loi autorisait donc l'IVG en cas d'urgence médicale (physique et mentale) pour la mère et ce, sur le territoire irlandais. Certains hôpitaux publics de la République étaient désormais habilités à prodiguer les soins. Comme le souligne Catherine Maignant

25. Edwige Nault, L'avortement en Irlande: 1983-2013..., p. 93.

26. Paul Curren, Kitty Holland, «Midwife Manager "Regrets" Using "Catholic Country" Remark to Savita Halappanavar», The Irish Times, 10 avril 2013.

27. Voir Irish Statute Book, Protection of Life during Pregnancy Act 2013, en ligne: http://www. irishstatutebook.ie/eli/2013/act/35/enacted/en/print. 
dans son article sur les relations entre l'Église et l'État depuis la création de l'État libre d'Irlande en 1922, le Protection of Life during Pregnancy Act fut un «tournant majeur dans l'histoire de l'Irlande». Elle insiste dans son travail sur «le passage d'un mode de représentation majoritaire fondé sur la loi naturelle à une vision du monde de plus en plus nettement humaniste ${ }^{28}$.

En décembre 2012, à l'initiative du gouvernement de coalition Fine GaelLabour, une Convention constitutionnelle (Constitutional Convention) avait été mise en place par le Parlement national irlandais, l'Oireachtas. Elle était composée d'un(e) président(e), de trente-trois membres choisis par les partis politiques et de soixante-six autres personnes tirées au sort parmi la population irlandaise inscrite sur les listes électorales. Sa mission était de s'exprimer sur des sujets de société; son rapport fut remis le 31 mars 2013. Il est à noter que la Convention se prononça à $79 \%$ en faveur d'une modification de la Constitution autorisant le «mariage pour tous», ce que le référendum du vendredi 22 mai 2015 confirma avec 62,1\% de voix favorables au mariage homosexuel. L'Irlande devint le premier pays au monde à légaliser le mariage gay par référendum. En matière de mœurs, comme le souligna le Premier ministre de l'époque, Enda Kenny, «les Irlandais pouvaient être des pionniers ». C'est d'ailleurs à son initiative que l'Assemblée citoyenne, de nature comparable à celle de la Convention constitutionnelle, fut installée en octobre 2016. Le Taoiseach avait probablement su tirer les enseignements de décennies de confusion et aussi de l'évolution des mentalités. L'expérience du passé devait servir à mieux affronter un sujet toujours tabou pour une part substantielle de la population irlandaise.

Obtenue en juillet 2013, la légalisation, même partielle, de l'avortement marqua sans aucun doute une rupture sociologique de grande ampleur et la mutation profonde des comportements se concrétisa par la forte diminution du pouvoir de l'Église sur les individus et l'État. Le discours politique touchant aux questions de morale sociale prenait alors de nouvelles formes au sein d'un nouvel espace - à la fois géographique, politique, culturel et social - lorsque fut mise en place l'Assemblée citoyenne inaugurée le 15 octobre 2016 au château de Dublin (Dublin castle).

L'Assemblée citoyenne succéda à la Convention constitutionnelle. Depuis sa création, elle est présidée par Mary Laffoy, juge à la Cour suprême, autorité reconnue pour avoir dirigé, de 1999 à 2003, la Commission relative aux abus sexuels à l'endroit des enfants ${ }^{29}$.

Soulignons le changement de nom qui s'opéra entre la première et la seconde assemblée. Même s'il convient de comprendre le mot anglais convention, utilisé dans l'expression Constitutional Convention, au sens latin d'assemblée, on peut remarquer que la nouvelle expression «the Citizens' Assembly» apparaissait déjà

28. Catherine Maignant, «Church and State in Ireland (1922-2013): Contrasting Perceptions of Humanity», Études irlandaises, n 39-2, 2014, p. 63.

29. Mary Carolan, «Tributes Paid to "Immense" Contribution of Ms Justice Mary Laffoy», The Irish Times, 16 juin 2017, p. 1. 
dans le programme du Fine Gael pour les élections législatives de février 2016. Nul doute que le gouvernement ait proposé un nom qui évoque d'emblée la démocratie participative pour des raisons stratégiques de communication avec les électeurs. Face à la méfiance grandissante à l'encontre du politique, phénomène quasi mondial, l'idée était séduisante et pleine de bon sens. Contrairement à la Convention constitutionnelle, l'Assemblée citoyenne ne comporte pas d'élus et ne compte que des citoyens tirés au sort sur les listes électorales. En souhaitant une participation plus directe des Irlandais, le gouvernement fit preuve d'une certaine ouverture vers davantage d'horizontalité, concept aujourd'hui en vogue, et souhaita innover dans les pratiques politiques. Bien que la mise en place de l'Assemblée citoyenne ne relevât pas de la spontanéité des électeurs, puisqu'elle avait été voulue par l'exécutif, on peut tout de même souligner la complémentarité des approches. Désormais, en effet, les citoyens de base jouent un rôle croissant puisqu'ils mènent un travail de réflexion en groupe sur des sujets aussi tabous que l'avortement. La parole citoyenne directe semble écoutée et participe de l'instauration d'un partenariat entre simples citoyens et élus.

En choisissant de confier à une Assemblée citoyenne le débat sur l'IVG, le gouvernement Fine Gael s'attira des reproches, voire des condamnations, venus du monde politique, y compris de certains élus ou journalistes progressistes qui voyaient, dans la création de l'Assemblée citoyenne, d'une part une remise en cause de la délégation de pouvoir accordée aux élus, et d'autre part une manière de repousser la tenue très attendue d'un référendum sur l'avortement ${ }^{30}$. S'agissait-il au contraire, pour le gouvernement, de mieux préparer le référendum, afin d'obtenir l'assurance de l'obtention d'une majorité de «oui» en faveur de l'IVG? Voulait-il éviter, comme ce fut le cas en 1992, d'amener les électeurs à se prononcer sur des libellés manquant de clarté? Cherchait-il à écarter du débat, autant que faire se pouvait, la hiérarchie catholique? Edwige Nault, dans une contribution récente de 2018, confirme «la perte de pouvoir de l'Église catholique sur la question de l'avortement» et s'interroge sur la remise en cause de la spécificité de l'identité irlandaise catholique qui avait vu le jour à la fin du XIX ${ }^{\mathrm{e}}$ siècle lorsque nationalisme et catholicisme s'entremêlaient pour combattre le pouvoir britannique ${ }^{31}$. L'État n'a-t-il pas souhaité, en proposant la concertation des citoyens au sein d'un groupe déterminé, encadrer ou orienter les discussions? Avec l'instauration de l'Assemblée citoyenne, le gouvernement témoigna de son souci d'améliorer la qualité des relations entre peuple et élus. La désignation de quatre-vingt-dix-neuf des cent membres ${ }^{32}$ de l'Assemblée fut unique dans le contexte irlandais et permit au gouvernement de s'assurer une part importante du soutien populaire. D'après sa

30. Una Mullaly, «Fight for Equal Rights Did Not End with Marriage Referendum », The Irish Times, 16 mai 2016, p. 14.

31. Edwige Nault, "Abortion in Ireland: From Religious Marginalisation to State Recognition», in Irishness on the Margins: Minority and Dissident Identities, Pilar Villar-Argáiz (dir.), Basingstoke, Palgrave Macmillan (New Directions in Irish and Irish American Literature), 2018, p. 129-130.

32. L'Assemblée comptait quatre-vingt-dix-neuf membres auxquels s'ajoutaient quatre-vingt-dix-neuf suppléants. 
présidente, Mary Laffoy, l'Assemblée constitue un véritable exercice de démocratie participative, le mode de choix de ses membres en étant le premier indice ${ }^{33}$. Le 9 août 2016, le cabinet du Premier ministre lança un appel d'offres pour choisir une société de sondage qui serait chargée de la sélection des membres de l'Assemblée citoyenne ${ }^{34}$. Choisis au hasard, ils devaient être représentatifs de la société irlandaise mais selon des critères définis: sexe, classe d'âge, catégorie socio-professionnelle, niveau d'instruction et répartition par régions ou comtés. Durant cinq week-ends, de novembre 2016 à avril 2017, l'Assemblée citoyenne se réunit pour faire des recommandations sur l'avenir du $8^{\mathrm{e}}$ amendement à la Constitution.

Dans son discours introductif du 29 juin 2017, prononcé à l'occasion de la présentation du rapport de l'Assemblée sur la question de l'avortement, Mary Laffoy insista sur le caractère tabou et clivant de la question au sein de la société irlandaise ${ }^{35}$. On remarquera d'ailleurs que, dans la plupart des pays qui ont dépénalisé l'avortement, la lenteur du processus a été semblable et que le simple fait d'entamer les discussions n'allait pas de soi. Cette première étape, une fois passée, permettait d'instaurer un débat, certes épineux, mais indispensable à l'obtention de nouveaux droits pour les femmes. En Irlande, comme ailleurs, les femmes préféraient souvent se taire puisque la société s'acharnait à les culpabiliser. Mary Laffoy et son équipe parvinrent à rendre le débat accessible à toutes et tous en offrant la possibilité à "madame ou monsieur Tout-le-Monde» de s'instruire, de comprendre et de se forger sa propre opinion.

La publicité des débats de l'Assemblée fut permise avec notamment l'enregistrement des délibérations par les caméras et l'utilisation des médias spécifiques comme Twitter. En regardant plus particulièrement le site Internet de l'Assemblée citoyenne ${ }^{36}$ et la chaîne YouTube Channel, les Irlandais pouvaient assister en direct aux séances publiques de l'Assemblée qui se réunissait, à chaque fois, au Grand Hotel de Malahide, près de Dublin. Sur les réseaux sociaux, les membres de l'Assemblée captèrent davantage l'attention du public et suscitèrent plus d'engagement de la part de leurs usagers. L'emploi des technologies actuelles allait donc servir à informer, à favoriser l'échange d'arguments et à cadrer les discussions. Le chercheur espagnol José Luis Dader, professeur de journalisme spécialisé en communication politique et en journalisme d'investigation, décrit les nouveaux outils comme un «immense répertoire d'instruments numériques d'approvisionnement ${ }^{37}$, d'échange et de discussion sur les messages politiques, [dont l'utilisation aurait été] impensable il y a deux décennies ${ }^{38}$. Il met toutefois

33. Mary Laffoy, "Chairperson's Introduction to the Citizens' Assembly and Summary ", in First Report and Recommendations of the Citizens' Assembly, 29 juin 2017, p. 1, on line: https://ptfs-oireachtas. s3.amazonaws.com/DriveH/AWData/Library3/CAdoclaid290617A_110031.pdf.

34. Stephen Collins, «Polling Firm to Choose Members of Abortion Group », The Irish Times, 9 août 2016, p. 2.

35. Mary Laffoy, "Chairperson's Introduction to the Citizens' Assembly and Summary», p. 1.

36. www.citizensassembly.ie/en

37. Par ce terme, on entend « ressources documentaires».

38. José Luis Dader, «La transformation de la politique sur Internet: de la politique-spectacle à la cyberdémocratie d'activisme citoyen", Netcom, vol. 26, nº 1-2, 2012, p. 18. 
en garde contre «les nouveaux outils [...] employés comme s'ils étaient d'anciens outils; c'est-à-dire, de nouvelles technologies pour des usages et avec des buts traditionnels ${ }^{39}$. La technologie transmédia permit au peuple irlandais de participer à l'Assemblée citoyenne en envoyant des milliers de contributions qui furent analysées et qui nourrirent le débat dans les deux camps. À l'achèvement des travaux substantiels de l'Assemblée en mai 2017, on ne compta pas moins de quatre-vingts heures d'auditions de spécialistes, de discussions et de délibérations qui s'étaient appuyées à la fois sur les nombreuses contributions citoyennes et sur celles émanant d'experts reconnus dans les milieux de la santé et du droit de la famille. Ces nombreux débats réalisèrent le souhait de Mary Laffoy de favoriser le processus démocratique. Comme le précise Jean-Pierre Gaudin, «la participation, tout comme la démocratie parlementaire, a besoin de scènes de discussion, de règles de débat, et d'évaluation de ses effets ${ }^{40}$. Les membres de l'Assemblée devaient préparer en amont le contenu des débats des séances suivantes en analysant les contributions citoyennes, en décryptant à l'avance les articles scientifiques et en lisant les publications des groupes de pression anti- et pro-avortement. L'approche pédagogique privilégiée permit aux participants de l'Assemblée de se familiariser à l'avance avec les concepts et les points de vue grâce, notamment, à l'emploi des outils de l'information et de la communication. Désormais, le débat ne se passait pas uniquement au sein des lobbies, des partis politiques, des syndicats, de l'Église catholique et des églises protestantes, comme ce fut le cas durant les différentes campagnes référendaires qui précédèrent celle des années 2017 et 2018 . L'Assemblée se devait de répondre non seulement aux attentes de ses membres mais aussi à celles d'une part grandissante du peuple irlandais qui souhaitait dorénavant choisir par elle-même en privilégiant une approche scientifique et objective, fondée sur les faits et l'analyse, et non sur les apports subjectifs provenant principalement de la hiérarchie catholique. En s'appuyant sur l'histoire de l'avortement en Irlande, en tenant compte des enseignements du passé et en accordant une place centrale aux témoignages des femmes ayant dû subir une IVG à l'étranger, l'Assemblée rompit avec les pratiques antérieures. La complexité du sujet imposa un travail soutenu des femmes et des hommes qui avaient été sélectionnés par Red C, l'organisme de sondage. Assiduité et application étaient de rigueur tant était grande la difficulté à libeller les textes sur lesquels ils devaient se prononcer, lors du dernier week-end de concertation en avril 2017. Par votes successifs, l'Assemblée citoyenne fit les recommandations suivantes à la commission parlementaire saisie du dossier ${ }^{41}$ :

- l'article 40.3.3 de la Constitution ne devrait pas du tout être conservé ( $87 \%$ d'avis favorables) ${ }^{42}$;

39. José Luis Dader, «La transformation de la politique sur Internet... », p. 18.

40. Jean-Pierre Gaudin, La démocratie participative, Paris, A. Colin, 2007, p. 15.

41. Mary Laffoy, "Chairperson's Introduction to the Citizens' Assembly and Summary», p. 3.

42. Lors du premier vote, la question posée fut la suivante: «Do you think Article $40.3 .3^{\circ}$ should be retained in full or not retained in full?». 79 personnes sur 91 présentes choisirent de ne pas maintenir le statu quo. 
- l'article 40.3.3 devrait être remplacé ou amendé (56\%) ${ }^{43}$;

- l'article 40.3.3 devrait être remplacé par une disposition constitutionnelle qui autoriserait le Parlement à légiférer en matière d'IVG et de droits de la mère et de l'enfant à naître $(57 \%)^{44}$.

On voit clairement, dans le résultat du troisième vote, le souhait de l'Assemblée d'opérer un transfert de compétences de la loi fondamentale à la loi ordinaire. L’Assemblée citoyenne ne devint-elle pas alors la meilleure alliée du pouvoir politique en place?

À l'issue des différents votes internes, on retiendra que $64 \%$ des membres de l'Assemblée se déclarèrent favorables à l'IVG et que, parmi eux, 48 \% étaient partisans de la pratique de l'IVG sans aucune restriction dans un délai légal de douze semaines. $44 \%$ optèrent même pour une durée de vingt-deux semaines de grossesse. Seuls $8 \%$ souhaitaient accorder une IVG sans aucune contrainte de délai ${ }^{45}$. L'Assemblée conseilla également d'améliorer la prévention en mettant en place des cours d'éducation sexuelle à l'école primaire et dans le secondaire.

En conclusion, l'engagement de l'Assemblée citoyenne sur une question aussi sensible fut sans aucun doute déterminant dans le résultat du référendum du 25 mai 2018, dans un pays où, après la révélation de divers scandales de l'Église, de plus en plus d'Irlandais se sentaient trahis par son discours, son langage et son dogmatisme. Moins d'un an après la diffusion des conclusions de l'Assemblée, plus de deux tiers des électeurs approuvaient la légalisation de l'IVG. Dans cette campagne, la première victoire n'avait-elle pas été obtenue lorsque l'Assemblée citoyenne avait préconisé, en juin 2017, une réforme libérale accordant le droit à l'avortement, sans restriction, jusqu'à douze semaines? Les centaines de témoignages de femmes, dont certaines relatèrent en public leur expérience récente de l'IVG médicamenteuse, confirmèrent que le statu quo n'était plus envisageable. Si, lors de la création de l'Assemblée, des voix nombreuses s'étaient opposées à sa mise en place, y compris dans le camp progressiste, invoquant une temporisation de l'État cherchant à nier l'urgence dans laquelle se trouvaient bon nombre de femmes ${ }^{46}$,

43. Lors du deuxième vote, la question était la suivante: «Do you think Article $40.3 .3^{\circ}$ should be repealed (i.e. deleted and not replaced) [option 1] or replaced or amended [option 2]?». 50 électeurs sur 91 votèrent pour l'option 2, 39 pour l'option 1 et 2 personnes s'abstinrent.

44. Lors du troisième vote, la question était la suivante: "How do you think Article $40.3 .3^{\circ}$ should be changed?». Les électeurs devaient choisir entre deux options: "Article $40.3 .3^{\circ}$ should be replaced with a constitutional provision that explicitly authorizes the Oireachtas to legislate to address termination of pregnancy, any rights of the unborn and any rights of the pregnant woman [option 1]» / Article 40.3. $3^{\circ}$ should be replaced or amended with a constitutional provision that directly addresses termination of pregnancy, any rights of the unborn and any rights of the pregnant woman [option 2]». Les options 1 et 2 obtinrent respectivement 51 et 38 voix. 3 personnes s'abstinrent.

45. Les électeurs favorables à l'IVG pouvaient voter pour l'une des trois options suivantes: le recours à l'IVG dans un délai de douze semaines (option 1), de vingt-deux semaines (option 2) et sans délai (option 3). Voir First Report and Recommendations of the Citizens' Assembly, 29 juin 2017, p. 36.

46. Ciarán d'Arcy, «Thousands Take Part in Pro-choice Rally», The Irish Times, 28 septembre 2016, p. 4. 
on notera que, à l'issue du scrutin, le rôle essentiel de l'Assemblée dans le débat et dans l'obtention du résultat fut unanimement reconnu dans les deux camps. À la suite des recommandations de l'Assemblée, les groupes de pression favorables à l'avortement, réunis sous la bannière de Together for Yes, prirent le relais sur le terrain et dans les nombreuses manifestations qui constituèrent, en elles-mêmes, de multiples assemblées citoyennes engagées au service des femmes. Dans la poursuite de notre travail de recherche, il conviendra de continuer à analyser l'impact de l'Assemblée citoyenne sur d'autres questions de société, notamment le vieillissement de la population ou la place du pays dans la lutte contre le réchauffement climatique. L'Assemblée parviendra-t-elle à garder un certain degré d'autonomie? Sera-t-elle aussi encline à la transparence et à l'ouverture? Ses membres ne vont-ils pas changer de statut en côtoyant la classe politique? Il nous faudra observer plus encore l'évolution de son fonctionnement afin de ne pas apporter de réponses prématurées à ces questions.

Christophe PoIRÉ

Université de Lorraine 